been recorded in the North of Scotland. However, there has been no prevalence study in this area since 1983.

Aims We undertook a new prevalence study of MS in Aberdeen City, and the Orkney and Shetland islands to: calculate age-gender specific prevalence rates; compare variations in age-gender standardised prevalence rates between areas and over time; calculate prevalence rates by MS sub-type, diagnostic criteria and to gather information on disability status.

Methods We used GP-practice records, hospital records and laboratory data for case ascertainment of patients alive and resident in the study area on prevalence day (24 September 2009), verified their diagnoses by reviewing medical records and included participants according to the research diagnostic criteria of Poser, McDonald 2001 and McDonald 2005. Information on disability was gathered from medical records and patient questionnaires. Prevalence rates and CIs were calculated assuming a Poisson distribution and standardised against the Scottish population (30 June 2009).

Results We found 590 patients in the combined study area (Aberdeen 442, Orkney 82, Shetland 66). Mean age was 52 years (SD $\pm 13)$, and the age-standardised male to female ratio was 1:2 (95\% CI 1.6 to 2.1). The standardised prevalence rate for the combined study area was 257 per 100000 (95\% CI 236 to 277), in Aberdeen City 237 per 100000 (95\% CI 214 to 257), in Orkney 421 per 100000 (95\% CI 329 to 512 ) and in Shetland 305 per 100000 (95\% CI 231 to 379). There were significant differences between Orkney and the other areas, and significant differences in the prevalence rates over time in Orkney and Shetland, but not for Aberdeen City. A relapse-remitting disease pattern was recorded in $50 \%$ of participants and $45 \%$ of patients had significant disability levels.

Conclusion The prevalence of MS has increased in the North of Scotland over the last 30 years, which may reflect methodological differences in studies over time, improved diagnostic methods, or a true increase in prevalence due to improved survival, higher incidence rates or as a result of migration. Currently Orkney has the highest MS prevalence rate in the world. New disability data could be used to plan health services in these communities.

\section{5-2.4 LOW-GRADE SYSTEMIC INFLAMMATION IN EARLY ADOLESCENCE PREDICTS SUBOPTIMAL BONE QUALITY IN LATE ADOLESCENCE: A PROSPECTIVE STUDY IN THE GENERAL POPULATION}

doi:10.1136/jech.2011.142976b.46

\footnotetext{
1,2 R Lucas, ${ }^{* 1,2} \mathrm{~T}$ Monjardino, ${ }^{1,2} \mathrm{E}$ Ramos, ${ }^{1,2} \mathrm{H}$ Barros. ${ }^{1}$ Department of Hygiene and Epidemiology, University of Porto Medical School, Porto, Portugal; ${ }^{2}$ Institute of Public Health of the University of Porto, Porto, Portugal
}

Introduction Early inflammatory changes may explain the negative impact of adiposity on bone acquisition during childhood. We aimed at estimating the effect of systemic inflammation during adolescence on forearm bone mineral density at 17 years-old.

Methods We used data from 377 girls born in 1990 and assessed at 13 and 17 years-old (EPITeen cohort). Adolescents were evaluated through physical examination, including height, weight and bone mineral density $(\mathrm{BMD})$ at the forearm using dual-energy $\mathrm{x}$-ray absorptiometry. Serum high-sensitivity $C$ reactive protein (CRP) was quantified (participants over $10 \mathrm{mg} / \mathrm{l}$ were excluded). Associations between CRP and BMD were quantified using linear regression. Coefficients were adjusted for gynaecologic age, weight and height, to minimise confounding by body size.

Results Median (25th-75th percentiles) CRP concentration increased from $0.2(0.1-0.5) \mathrm{mg} / \mathrm{l}$ at 13 to $0.6(0.2-1.7)$ at 17 yearsold. Mean (SD) BMD was $0.362(0.058) \mathrm{g} / \mathrm{cm}^{2}$ at 13 and 0.437 $(0.052) \mathrm{g} / \mathrm{cm}^{2}$ at 17 . Adolescents in the upper quarter of CRP at 13 had similar adjusted mean $\mathrm{BMD}$ at that age but significantly lower $\mathrm{BMD}$ at 17 years-old when compared to those in the lowest quarter
(-0.024, 95\% CI -0.040 to -0.007$)$. Additionally, girls in the two highest quarters of CRP variation had significantly lower BMD at 17 when compared to the lowest quarter $(-0.016,95 \% \mathrm{CI}-0.031$ to -0.001 and $-0.027,95 \%$ CI -0.043 to -0.010 , respectively).

Conclusion Systemic inflammation in early adolescence and its increase during follow-up, predicted lower bone quality in late adolescence, providing evidence that the negative association between obesity and bone accrual is probably mediated by lowgrade inflammation.

\section{5-2.5 LIFE COURSE BMI AND RISK OF KNEE OSTEOARTHRITIS AT AGE 53: EVIDENCE FROM THE 1946 BRITISH BIRTH COHORT STUDY}

doi:10.1136/jech.2011.142976b.47

${ }^{1} \mathrm{~A}$ Wills, ${ }^{*}{ }^{1} \mathrm{~S}$ Black, ${ }^{2} \mathrm{R}$ Coppack, ${ }^{1} \mathrm{R}$ Cooper, ${ }^{1} \mathrm{R}$ Hardy, ${ }^{3} \mathrm{C}$ Cooper, ${ }^{1} \mathrm{D}$ Kuh. ${ }^{1} \mathrm{M} R \mathrm{C}$ Unit for LHA, University College London, London, UK; ${ }^{2}$ DMRC Headley Court, Epsom, Surrey, UK; ${ }^{3}$ MRC Lifecourse Epidemiology Unit, University of Southampton, Southampton, UK

Introduction We examined how body mass index (BMI) over the lifecourse influences the risk of later life knee osteoarthritis $(\mathrm{OA})$, for example, whether knee OA risk accumulates with prolonged exposure to high BMI or whether later rather than earlier adult life is the key period of exposure.

Methods A population-based birth cohort study of 3035 men and women who underwent a clinical examination for knee $\mathrm{OA}$ at age 53. BMI was measured 10 times from 2 to 53 years. Analyses were stratified by gender and adjusted for occupation and activity levels.

Results The prevalence of knee OA was higher in women than men-12.9\% ( $n=194)$ vs $7.4 \% \quad(n=108)$. In men, the association between BMI and knee OA was apparent at age $20(p=0.038)$ and remained until 53 yrs (OR per z-score: 1.38 ; $95 \%$ CI 1.11 to 1.71 ). In women, there was evidence for an association at $15 \mathrm{yrs}(\mathrm{p}=0.003)$; this became stronger through adulthood- at age 53 the OR was 1.89 (CI 1.59 to 2.24) per z-score increase in BMI. A structured modelling approach to disentangle the way in which BMI over life influenced knee OA risk suggested that in women, prolonged exposed to high BMI throughout adulthood carried the highest risk, while in men, it was exposure in mid adulthood that explained most of the risk.

Conclusion Our study suggests that, particularly in women, the duration of exposure to high BMI in adulthood is important in explaining knee OA risk, and that these associations originate from weight gain in childhood and adolescence.

\section{5-2.6 LAG EFFECTS OF INCOME INEQUALITY ON TOOTH LOSS: A MULTILEVEL STUDY OF US ADULTS}

doi:10.1136/jech.2011.142976b.48

${ }^{1,2}$ E K Delgado-Angulo, ${ }^{*}$ E Bernabé. ${ }^{1}$ Department of Epidemiology and Public Health, University College London, London, UK; ${ }^{2}$ Departamento Académico de Odontología Social, Universidad Peruana Cayetano Heredia, Lima, Peru, ${ }^{3}$ Institute of Dentistry, Barts and The London, Queen Mary University of London, London, UK

Introduction Income inequality has detrimental effects on health and oral health. However, this effect is doubtfully instantaneous and most studies have measured both income inequality and health contemporaneously. This study examined the association between state-level income inequality and tooth loss among adults in the United States, under different assumptions about lag periods.

Methods This study pooled individual data from the 2008 Behavioural and Risk Factor Surveillance System and state-level data from the US Census Bureau. The Behavioural and Risk Factor Surveillance 
System collected information on demographic characteristics (age, sex, race/ethnicity and marital status), socioeconomic position (education and annual household income) and tooth loss, which was self-reported on a 4-point scale (none, 1 to 5, 6 or more but not all, and all teeth). Income inequality at state level was measured with the Gini coefficient, based on household income for 1979, 1989 and 1999, respectively. Two-level ordered logit models with individuals nested within states were used to test the association of state Gini coefficient at different lag periods with tooth loss after adjustment for state median household income and individuals' characteristics.

Results In the fully adjusted models, state Gini coefficient was significantly associated with self-reported tooth loss when testing lag periods of 10 - (OR: $1.18,95 \%$ CI 1.05 to 1.32 ) and 20 years (OR $1.16,95 \%$ CI 1.02 to 1.32 ). However, no association was found when testing the 30-year lag period (OR 1.12, 95\% CI 0.92 to 1.35).

Conclusion This study suggests that state income inequality has stronger effects on self-reported tooth loss up to 20 years later. 